Le chapitre sur la France fait connaître les résultat d'une législation que les auteurs souhaitent de tous leur vœux voir adopter au Québec. Assez curieusement, ils évoquent les performances récentes (cinq dernières années) dont les grandes entreprises hexagonales feraient la preuve. Or, pour ma part, j'entends parler d'essaimage au pays d'Astérix depuis au moins vingt ans. Rien de neuf donc sous le soleil de la France et de Navarre. Ici sont fournis les exemples de grandes entreprises comme Air France, Aventis, EDF, La poste, Renault, Saint-Gobain et autres Société générale. Rien à voir avec la France profonde. Les auteurs ne parlent effectivement pas de régions périphériques en signalant ces exemples regroupés à l'intérieur de l'Association pour le développement de l'initiative et l'entrepreneuriat chez les salariés.

En conclusion, sous la forme - encore une fois d'un tableau récapitulatif se trouvent présentés les principaux facteurs de succès de l'essaimage parmi lesquels on a : l'engagement des dirigeants, le système d'aide et de soutien de l'organisation essaimante, la conception conjointe du projet, etc.

Ce volume ne manquera pas d'intéresser tout ceux qui, en région, gravitent autour des PME innovantes et dynamiques, lesquelles, si elles ne sont pas suffisamment nombreuses, existent bel et bien. Leur exemple ne peut qu'être utile pour ceux qui ont à cœur le dynamisme de leur région. Écrit par des auteurs crédibles et convaincus (par le fait même convaincants), cet ouvrage demeure fidèle autant par la forme que par le fond à sa collection. L'accent est placé exclusivement sur la pratique, le tout accompagné de peut-être un trop grand nombre de tableaux où sont dressés, sous la forme de listes d'épicerie, des éléments parfois redondants qui se veulent une synthèse des sections concernées. Au lecteur de s'attarder à ce qu'il juge utile pour lui.

\section{Claude Dupuis et Antjie Burmeister (sous la direction de), Entreprises et territoires : les nouveaux enjeux de la proximité Paris, La documentation Française, 2003, 134 p.}

Mieux vaut être la tête d'un poulet que la queue d'une vache; en d'autre mots, il est préférable d'être le pa- tron d'une petite entreprise que le dernier des subalternes d'une grande entreprise. Ce dicton chinois est fourni dans un encadré sur un parc scientifique de Taiwan où il est question ici aussi d'essaimage. Les auteurs de ce chapitre qui, on l'imagine bien, n'ont pas lu l'ouvrage précédent, ont cru bon de fournir la traduction du mot essaimage : spin off. C'est d'ailleurs le seul rapprochement que l'on puisse faire avec l'ouvrage ci-haut présenté, car les 19 auteurs de ce volume, de dimension restreinte mais très dense, destinent leurs contributions, réparties en six chapitres, aux lecteurs universitaires. Les agents de développement économique sentant le besoin de rafraîchir leurs notions d'économie régionale, à défaut d'y trouver des informations susceptibles de faciliter leur tâches quotidiennes, y puiseront néanmoins des considérations conceptuelles et factuelles en mesure de répondre à leur curiosité intellectuelle. Par le fait même, ils pourraient en arriver à mieux comprendre l'évolution de leur milieu d'intervention.

Comme l'indique le sous-titre de l'ouvrage, les textes se regroupent sous le terme d'"économies de la proximité $»$. On remarquera le recours au pluriel, car on se rapporte à diverses situations. Utilisée au singulier, l'expression se rapporte aux actions prises par la gouvernance territoriale, avec une attention toute particulière aux relations hors marché et donc autant aux institutions formelles (organisations publiques) qu' informelles (règles, habitudes comportements...). Comme il est indiqué dans un chapitre sur les réseaux de transport et de télécommunication, l'efficacité d'un système productif repose sur la qualité des interactions entre acteurs, qui nécessite moins une proximité géographique, ou une bonne accessibilité entre eux, qu'une proximité organisationnelle facilitant la mise en place des mécanismes de coordination.

Cet aréopage d'auteurs, pour la plupart associés à une université ou à un centre de recherche, s'est donné pour tâche l'identification des avantages que les acteurs économiques et leurs territoires retirent de leur proximité ainsi que ses limites. Sans trop s'engager dans les développements théoriques, ils tirent profit d'une somme variée d'études de terrain en vue d'en faire ressortir les enseignements pour les fins des politiques publiques. Puisqu'il est abondamment question de gouvernance territoriale, mentionnons qu'elle se définit ici comme le processus institutionnelorganisationnel de construction d'une mise en compa- 
tibilité de différents modes de coordination entre acteurs géographiquement rapprochés. Pour la rendre opérationnelle, il est bien indiqué qu'il importe d'obtenir, de la part des acteurs concernés, la mise en synergie de leurs trajectoires respectives de façon à favoriser l'émergence de l'intérêt commun. Chacun des chapitres comprend des encadrés facilitant la mise en évidence d'exemples concrets. La technopole de Lyon-Guerland et le site de Lacq en Aquitaine servent ici d'illustrations.

Le chapitre sur l'organisation en réseau s'avère particulièrement intéressant pour les lecteurs d'Organisations et territoires. Ses auteurs se demandent dans quelle mesure les réseaux institutionnels jouent le jeu de la proximité géographique. En considérant que cette dernière favorise le processus de développement technologique, ils cherchent à montrer si les réseaux peuvent vraiment favoriser les effets de synergie. Cependant, c'est le dernier chapitre sur les réseaux de transport et de télécommunication qui m'est apparu le plus intéressant. Ces deux types de réseaux non relationnels sont ici envisagés comme des moyens alternatifs et complémentaires de coordination plutôt que sous la forme classique des effets des infrastructures sur le développement économique. Les auteurs remettent en cause les politiques publiques largement fondées sur la logique mécanique de l'effet structurant des infrastructures de transport. Ils semblent être conscients que l'autoroute Félix-Leclerc, reliant Montréal à Québec via Trois-Rivières, n'a eu aucun impact économique sur l'ex-capitale mondiale du papier. Tout comme ils semblent vouloir dire aux Saguenayens que, s'il serait bien qu'une autoroute remplace l'actuelle route 175 , ils ne doivent pas s'illusionner sur les éventuelles retombées économiques susceptibles d'en résulter.

C'est dans ce chapitre que l'on trouve une curieuse définition du capital social puisée dans une thèse récente où le jeune doctorant, de toute évidence, n'a pas subi la même influence que les écrits de Pierre Bourdieu ont eu sur nos sociologues. Qu'on en juge : «Le capital social est constitué de l'ensemble des structures et des équipements du système de santé et d'éducation, des services culturels et de loisirs..." , etc. Exit la cohésion sociale. Pas grave. L'important, c'est que plusieurs trouveront ici et là dans cet ouvrage matière à réflexion.

\section{Jacques de Courson \\ Les élus locaux : qui sont-ils ? Que ont-ils ? Comment travaillent-ils? Paris, Éditions d'organisation, 2001, 215 p.}

Voilà un titre qui ne manquera pas d'accrocher les Québécois à l'heure où les nouvelles Conférences régionales des élus se mettent en branle. Oui, l'acronyme CRÉ va devenir bientôt très familier pour tous ceux qui s'intéressent à la politique locale ou régionale. Leur implantation ne s'est pas faite sans soulever bon nombre d'interrogations, par exemple sur le rôle qui sera désormais dévolu à la société civile. Cependant, il faut en convenir, ce n'est pas depuis l'avènement d'un nouveau gouvernement à la barre du Québec que les élus locaux ont pris du gallon. Le rôle exercé par les maires de petites et moyennes municipalités dans le développement local fait l'objet d'une attention certaine depuis déjà quelques années. Alors, on comprendra la pertinence des questions soulevées dans le titre de cet ouvrage. En effet, qui sontils ces maires et comment remplissent-ils leur mandat eu égard au développement économique ? Ces vingt dernières années, décentralisation oblige, la gestion municipale a dû évoluer. En effet, la mission économique à l'échelon d'un regroupement de villages ou d'une municipalité tend à prendre davantage d'importance.

Dans ces conditions, l'ouvrage de notre collègue Jacques de Courson, de l'Université Paris XII, ne peut passer inaperçu. Mais oui, pour le lecteur québécois, il y a un « mais » : l'ouvrage concerne la réalité hexagonale uniquement. Cependant, ce « mais » n'enlève pas une grande partie de l'intérêt de cet ouvrage. Par exemple, avec le premier chapitre intitulé «Qui sont nos élus locaux? », les Québécois reconnaitront de toute évidence beaucoup de leurs propres élus. Par ailleurs, sur la façon dont ils sont élus (chap. 2), force est d'admettre que l'on se trouve dans un autre monde. Et si ce qui se rapporte au statut rattaché à la fonction d'élu local (chap. 3) colle dans une certaine mesure à tout contexte, on conviendra que le cumul des mandats représente bien une réalité franco-française. En ce qui regarde la vie quotidienne de monsieur le maire (chap.4), encore une fois les exigences de la fonction outre-mer ne sont pas vraiment différentes de 